Proceedings of the XXXVIII International School and Conference on the Physics of Semiconductors "Jaszowiec" 2009

\title{
ZnCoO Films by Atomic Layer Deposition - Influence of a Growth Temperature on Uniformity of Cobalt Distribution
}

\author{
M.I. Łukasiewicz ${ }^{a, *}$, B. Witkowski ${ }^{b}$, M. Godlewski ${ }^{a, b}$, E. GuzIeWicz $^{a}$, M. SAWicki ${ }^{a}$, \\ W. PAszkowicz ${ }^{a}$, E. Łusakowska ${ }^{a}$, R. JAkiela ${ }^{a}$, T. Krajewski ${ }^{a}$, I.A. Kowalik ${ }^{a}$ \\ AND B.J. KOWALSKI ${ }^{a}$ \\ ${ }^{a}$ Institute of Physics, Polish Academy of Sciences, al. Lotników 32/46, 02-668 Warsaw, Poland \\ ${ }^{b}$ Dept. of Mathematics and Natural Sciences, College of Science \\ Cardinal S. Wyszyński University, Dewajtis 5, 01-815 Warsaw, Poland
}

\begin{abstract}
We report on the structural, electrical and magnetic properties of $\mathrm{ZnCoO}$ thin films grown by atomic layer deposition method using reactive organic precursors of zinc and cobalt. As a zinc precursor we applied either dimethylzinc or diethylzinc and cobalt(II) acetyloacetonate as a cobalt precursor. The use of these precursors allowed us the significant reduction of a growth temperature to $300^{\circ} \mathrm{C}$ and below, which proved to be very important for the growth of uniform films of $\mathrm{ZnCoO}$. Structural, electrical and magnetic properties of the obtained $\mathrm{ZnCoO}$ layers will be discussed based on the results of secondary ion mass spectroscopy, scanning electron microscopy, energy dispersive spectroscopy, X-ray diffraction, atomic force microscopy, Hall effect and SQUID investigations.
\end{abstract}

PACS numbers: 68.55.Ln, 68.55.Nq, 78.66.Hf, 81.15.Kk

\section{Introduction}

Diluted magnetic semiconductors (DMS) have attracted much attention as potential materials for spintronic devices. ZnTMO, with TM standing for transition metals (Co, Mn, Fe, etc.), is one of the promising DMS materials [1-3]. Theoretical calculations have shown that ferromagnetic state can be obtained at room temperature (RT) in $p$-type $\mathrm{ZnMnO}$ [1], but also it may be possible in $n$-type $\mathrm{ZnO}$ when doped with cobalt $[2,3]$. In fact, ferromagnetic ordering at $\mathrm{RT}$ in $\mathrm{ZnCoO}$ was reported by several groups. However, the origin of the ferromagnetism in $\mathrm{ZnCoO}$ is not clear. It is more likely due to metal accumulations and foreign phases, rather than volume properties of samples. Thus, confusing reports on their magnetic properties can be partly related to inclusions of foreign phases and metal accumulations $[4,5]$.

The objective of this investigation is to present and discuss the results of structural, magnetic, and electrical measurements made on $\mathrm{ZnCoO}$ thin films obtained at low temperature (LT) growth conditions by the atomic layer deposition (ALD) method.

\section{Experimental}

The $\mathrm{ZnCoO}$ thin films were grown using the ALD technique [6]. We used organic precursors: dimethylzinc and

\footnotetext{
* corresponding author; e-mail: mluk@ifpan.edu.pl
}

diethylzinc as a zinc precursors, and cobalt(II) acetyloacetonate as a cobalt precursor. Deionized water was used as an oxygen precursor. These highly reactive precursors are sequentially introduced to the growth chamber, so they meet only at a surface of a grown film. The use of these precursors allowed us the significant reduction of a growth temperature to $300^{\circ} \mathrm{C}$ and below.

Precursors' temperatures were the following: DMZn $30^{\circ} \mathrm{C}, \mathrm{DEZn}-\mathrm{RT}, \mathrm{Co}(\mathrm{acac})_{2}-150^{\circ} \mathrm{C}$, and water $30^{\circ} \mathrm{C}$. Substrate temperature (we used sapphire, GaAs, $\mathrm{Si}$, glass) ranged between $160^{\circ} \mathrm{C}$ and $300^{\circ} \mathrm{C}$.

\section{Results}

The structural characterization was carried out by X-ray diffraction (XRD) method. XRD spectra for films grown at two different temperatures are shown in Fig. 1. The XRD measurements show a polycrystalline nature of $\mathrm{ZnCoO}$ layers. In all the cases we observed the diffraction maxima related to (10.0), (00.2) and (10.1) crystallographic orientations. The crystalline structure was significantly better for films grown at lower temperature $\left(160^{\circ} \mathrm{C}\right)$ than for the one grown at $300^{\circ} \mathrm{C}$. The surface morphology of $\mathrm{ZnCoO}$ films was investigated with atomic force microscopy (AFM) and scanning electron microscopy (SEM). Root mean square (RMS) value of roughness of the films ranged between $1.28 \mathrm{~nm}$ and $22 \mathrm{~nm}$, depending on the film thickness and growth temperature (RMS increases with the thickness but decreases with increasing temperature). 


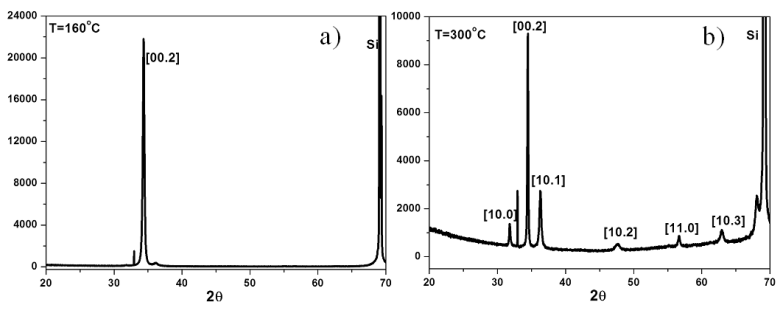

Fig. 1. XRD spectra for $\mathrm{ZnCoO}$ films grown at different two temperatures: (a) $160^{\circ} \mathrm{C}$, (b) $300^{\circ} \mathrm{C}$.

SEM images taken at relatively high magnification are shown in Fig. 2 for two growth temperatures $300^{\circ} \mathrm{C}$ (a) and $160^{\circ} \mathrm{C}$ (c). SEM images show flake-like microstructure of $\mathrm{ZnCoO}$ films grown with a few percent Co fraction.

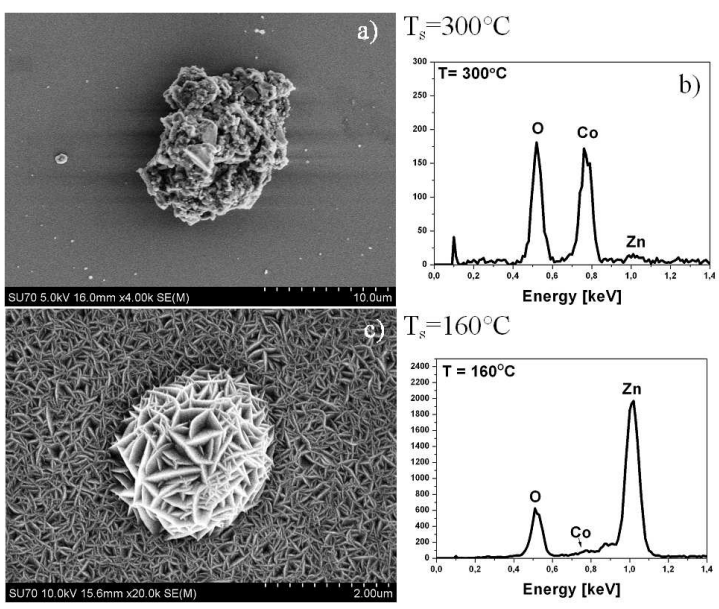

Fig. 2. SEM images (left column) and EDS (right column) spectra for $\mathrm{ZnCoO}$ films grown at two different temperatures: (a), (b) $300{ }^{\circ} \mathrm{C}$, (c), (d) $160^{\circ} \mathrm{C}$.

In Fig. 2a,c we show islands formed on a surface of the studied films (such islands appear very sporadic). The chemical composition of these islands was determined by using the energy dispersive spectroscopy (EDS) method. Results of EDS measurements are shown in Fig. $2 \mathrm{~b}$ and $\mathrm{d}$. In the case of a higher growth temperature these islands consist of a $\mathrm{CoO}$ foreign phase, which is not the case of the samples grown at lower temperature. Maps of the EDS spectra taken from flat surface area indicated fairly in-plane uniform Co distribution for both types of the $\mathrm{ZnCoO}$ films.

Depth uniformity of $\mathrm{Co}$ concentration in $\mathrm{ZnCoO}$ films was studied with secondary ion mass spectroscopy (SIMS). SIMS investigations showed high homogeneity of the $\mathrm{ZnCoO}$ layers, when grown with the optimized ALD growth parameters. To optimize these parameters we tested several sequences of elementary ALD cycles and times of pulses of cobalt precursor. We obtained the best results for the following sequences of the ALD cycles eighty cycles to form layers of $\mathrm{ZnO}$ followed by five cy- cles to form layers of $\mathrm{CoO}$. Films grown with thinner $\mathrm{ZnO}$ "spacers" were less uniform and showed worse crystallographic structure.

Figures 2a and c illustrate difficulties in obtaining more ordered films. We found two effects related to an increasing temperature of the process. First, Co fraction in $\mathrm{ZnCoO}$ increases (from $1.4 \%$ to 5.5 atomic \%) in the samples grown at higher temperature, even though precursor pulses were the same. Second, the growth mode depends on a growth temperature. It is more 3D-like for samples grown at low temperature (see Fig. 2c), with columns (flakes-like) of mixed orientation, as deduced from the XRD investigations. To obtain films with a better crystallographic structure it was necessary to reduce Co fraction to say below 5\%, lower growth temperature and to introduce relatively thick $\mathrm{ZnO}$ "spacers" in the ALD growth cycles.

The Hall measurements conducted on the samples of $\mathrm{ZnCoO}$ layers showed that the electron concentration varied between $10^{15} \mathrm{~cm}^{-3}$ and $10^{19} \mathrm{~cm}^{-3}$, depending on the growth temperature, Co fraction and time of application of Co-precursor (Fig. 3). Important observation is that we can achieve high $n$ doping in $\mathrm{ZnCoO}$ films. On the other hand, we observed a surprising anti-correlation between length of Co precursor pulses and carrier concentration and mobility (see Fig. 3).

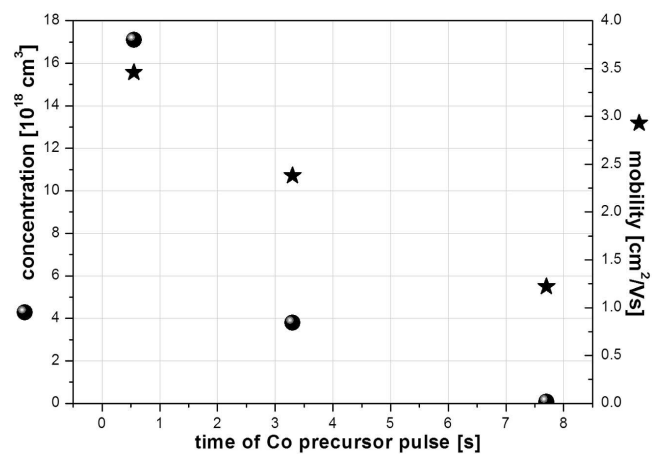

Fig. 3. Anti-correlation between time of Co precursor pulses and sample conductivity (electron concentration and mobility).

After setting optimal conditions for $\mathrm{ZnCoO}$ deposition we performed magnetization investigations using SQUID magnetometer. Films of $\mathrm{ZnCoO}$ with homogeneous $\mathrm{Co}$ distribution, with Co fraction below $5 \%$, and these which were grown at LT show paramagnetic properties at RT. Nonuniform films show multi-phase signals in SQUID measurements.

\section{Conclusions}

It is important to underline that $\mathrm{ZnCoO}$ turned out to be by far more difficult to be grown as uniform films (as compared e.g. to $\mathrm{ZnMnO}$ ). Anyway, by optimizing sequences of the ALD cycles and the growth temperature 
we have grown uniform $\mathrm{ZnCoO}$ films showing only paramagnetic response in the SQUID measurements. We also observed that high $n$-type doping is possible in $\mathrm{ZnCoO}$.

\section{Acknowledgments}

This work was supported by FunDMS ERC Advanced Grant Research.

\section{References}

[1] T. Dietl, H. Ohno, F. Matsukura, Science 287, 1019 (2000).
[2] K. Sato, H. Katayama-Yoshida, Jpn. J. Appl. Phys. 39, L555 (2000).

[3] K. Sato, H. Katayama-Yoshida, Jpn. J. Appl. Phys., Part 2 40, L334 (2001).

[4] A. Wójcik, K. Kopalko, M. Godlewski, E. Guziewicz, R. Jakieła, R. Minikayev, W. Paszkowicz, Appl. Phys. Lett. 89, 051907 (2006).

[5] A. Wójcik, M. Godlewski, E. Guziewicz, K. Kopalko, R. Jakieła, M. Kiecana, M. Sawicki, M. Guziewicz, M. Putkonen, L. Niinistö, Y. Dumont, N. Kelner, Appl. Phys. Lett. 90, 082502 (2007).

[6] T. Suntola, Mater. Sci. Rep. 4, 261 (1989). 